\title{
The 2000 revision of the joint UK/US geomagnetic field models and an IGRF 2000 candidate model
}

\author{
S. Macmillan ${ }^{1}$ and J. M. Quinn ${ }^{2}$ \\ ${ }^{1}$ British Geological Survey, Murchison House, West Mains Road, Edinburgh, EH9 3LA, Scotland \\ ${ }^{2}$ United States Geological Survey, MS 966, Federal Center, Denver, CO 80225-0046, U.S.A.
}

(Received February 21, 2000; Revised June 27, 2000; Accepted July 8, 2000)

\begin{abstract}
The method of derivation of the joint UK/US spherical harmonic geomagnetic main-field and secular-variation models is presented. Early versions of these models, with the main field truncated at degree 10, are the UK/US candidates for the IGRF 2000 model. The main-field model describes the Earth's magnetic field at the 2000.0 epoch, while the secular-variation model predicts the evolution of this field between 2000.0 and 2005.0. A revised 1995.0 main-field model was also generated. Regional models for the continental US, Alaska and Hawaii were also produced as a by-product of the UK/US global modelling effort.
\end{abstract}

\section{Introduction}

The World Magnetic Model for epoch 2000 (WMM2000) is produced jointly by the British Geological Survey (BGS) in Edinburgh, Scotland and the U.S. Geological Survey (USGS), in Denver, Colorado on behalf of the UK Hydrographic Office in Taunton, England and the National Imagery and Mapping Agency in Reston, Virginia, USA. The WMM has a wide range of applications in commercial and military navigational and attitude/heading reference systems, geophysical prospecting, and basic research. It may be found as a piece of software in many Global Positioning System (GPS) receivers, on ships, and in aircraft, missiles, and satellites. This model is used to calibrate compass roses and to assign runway designations at airports. The World Hydrographic Office in Monaco has also adopted it as its official model for international ship navigation on the world's oceans.

The model is intended to characterise only that portion of the Earth's magnetic field originating from the Earth's core. Consequently, magnetic fields originating from sources within the Earth's crust, ionosphere and magnetosphere have been removed to the extent that it is possible to do so. WMM2000 comprises a main-field model for epoch 2000.0 and a predictive secular-variation model for the period 2000.0 to 2005.0. This model will be replaced in 5 years by WMM2005. A revised 1995.0 epoch model has also been derived.

In deriving WMM2000 the main field and the secular variation are modelled separately. This strategy means that different data can be used for main-field and secular-variation modelling, thereby optimising their different strengths. As it is a model intended for navigation it is necessary that it gives magnetic field values for dates for some time into the future. Its ability to predict secular variation is therefore very important. Not all data types are suitable for secularvariation prediction and, in addition, some data types are

Copy right (c) The Society of Geomagnetism and Earth, Planetary and Space Sciences (SGEPSS); The Seismological Society of Japan; The Volcanological Society of Japan; The Geodetic Society of Japan; The Japanese Society for Planetary Sciences. better than others for minimising contributions from crustal and external field variations. BGS is primarily responsible for secular-variation modelling and USGS for main-field modelling. However, during the modelling process there is feedback between the two modelling groups.

Three 5-year average secular-variation models were required for the production of the WMM2000 valid for the periods 1990.0-1995.0, 1995.0-2000.0 and 2000.0-2005.0 respectively. The first two models are used to reduce the main-field data to epoch and the last model allows computation of main-field values after 2000.0. The secular-variation models are based mainly on geomagnetic observatory data.

The main-field model for 2000.0 is based mainly on scalar data from the Danish satellite Ørsted. Vector data in the magnetic equatorial area are provided by a revised version of a model produced for WMM-95, reduced to epoch.

\section{The Mathematical Model}

In a source-free region near the surface of the Earth the magnetic field $\mathbf{B}$ is the negative gradient of a scalar potential, i.e.

$$
\mathbf{B}=-\nabla V(r, \theta, \lambda, t)
$$

which satisfies Laplace's equation

$$
\nabla^{2} V(r, \theta, \lambda, t)=0
$$

where the spherical coordinates $(r, \theta, \lambda)$ correspond to the radial distance from the centre of the Earth, the geocentric colatitude $\left(90^{\circ}\right.$-latitude $)$ and the longitude, and $t$ is the time. A solution to Laplace's equation in spherical coordinates is

$$
\begin{aligned}
V(r, \theta, \lambda, t)= & a \sum_{n=1}^{n i_{\max }}\left(\frac{a}{r}\right)^{n+1} \sum_{m=0}^{n}\left(g_{n}^{m}(t) \cdot \cos m \lambda\right. \\
& \left.+h_{n}^{m}(t) \cdot \sin m \lambda\right) \cdot P_{n}^{m}(\theta) \\
& +a \sum_{n=1}^{n e_{\max }}\left(\frac{r}{a}\right)^{n} \sum_{m=0}^{n}\left(q_{n}^{m}(t) \cdot \cos m \lambda\right. \\
& \left.+s_{n}^{m}(t) \cdot \sin m \lambda\right) \cdot P_{n}^{m}(\theta)
\end{aligned}
$$


where $a$ is the mean radius of the Earth $(6371.2 \mathrm{~km}) ; g_{n}^{m}(t)$ and $h_{n}^{m}(t)$ are the internal Gauss coefficients at time $t ; q_{n}^{m}(t)$ and $s_{n}^{m}(t)$ are the external Gauss coefficients at time $t$ and $P_{n}^{m}(\theta)$ are the Schmidt-normalised associated Legendre functions of degree $n$ and order $m$. The Gauss coefficients are functions of time and for navigation purposes the internal ones are assumed to vary at a constant rate for the next five-year period:

$$
\begin{array}{ll}
g_{n}^{m}(t)=g_{n}^{m}+\dot{g}_{n}^{m} \cdot\left(t-t_{0}\right) & t_{0} \leq t \leq t_{0}+5 \\
h_{n}^{m}(t)=h_{n}^{m}+\dot{h}_{n}^{m} \cdot\left(t-t_{0}\right) & t_{0} \leq t \leq t_{0}+5
\end{array}
$$

where $g_{n}^{m}$ and $h_{n}^{m}$ are the main-field coefficients for the base epoch of the model, $t_{0}$, and $\dot{g}_{n}^{m}$ and $\dot{h}_{n}^{m}$ are the annual secularvariation coefficients for the 5-year period following the base epoch. Predictions of the main field for more than 5 years into the future are not sufficiently accurate for general navigation purposes and for this reason models and charts are revised every five years. The first three external coefficients are given by:

$$
\begin{aligned}
& q_{1}^{0}(t)=q_{1}^{0}+q_{1, D s t}^{0} \cdot \operatorname{Dst}(t) \\
& q_{1}^{1}(t)=q_{1}^{1}+q_{1, D s t}^{1} \cdot \operatorname{Dst}(t) \\
& s_{1}^{1}(t)=s_{1}^{1}+s_{1, D s t}^{1} \cdot \operatorname{Dst}(t) .
\end{aligned}
$$

The Dst index is taken to be a characterisation of ring current activity. The remaining external coefficients are assumed not to vary with time over the time span of the input data for the model. Magnetic induction within the Earth generated by the ring current is taken into account through the $g_{1}^{0}$ internal Gauss coefficient:

$$
g_{1}^{0}(t)=g_{1}^{0}+g_{1, q}^{0} \cdot q_{1}^{0}(t) .
$$

The maximum degree for the main field, $n i_{\max }$, is set at 12 ; for the external field, $n e_{\max }=5$ and for the secular variation of the main field, $n i_{\max }=8$. The main-field maximum degree of 12 includes contributions of approximately $87 \%$ core field and $13 \%$ crustal field. Degree 13 main-field model coefficients would contain a contribution of approximately $48 \%$ crustal field and consequently, degree 13 is considered to be the beginning of a transition region from core dominance to crustal dominance. Normally, as a result of experiences with Magsat data, only degree 1 external field terms are considered. However, for Ørsted data several external field coefficients from degrees 2 to 5 , including $q_{5}^{1}$ and $s_{5}^{3}$, were found to be greater than $1 \mathrm{nT}$, and so were considered significant. The secular variation cannot be modelled to the same maximum degree as the main field because there are insufficient data. The equations for observations of the magnetic field are

$$
\begin{aligned}
B_{r}= & -\frac{\partial V}{\partial r}=\sum_{n=1}^{12}(n+1)\left(\frac{a}{r}\right)^{n+2} \sum_{m=0}^{n} \\
& \cdot\left(g_{n}^{m} \cdot \cos m \lambda+h_{n}^{m} \cdot \sin m \lambda\right) \cdot P_{n}^{m}(\theta) \\
& -\sum_{n=1}^{5} n\left(\frac{r}{a}\right)^{n-1} \sum_{m=0}^{n} \\
& \cdot\left(q_{n}^{m} \cdot \cos m \lambda+s_{n}^{m} \cdot \sin m \lambda\right) \cdot P_{n}^{m}(\theta) \\
B_{\theta}= & -\frac{1}{r} \frac{\partial V}{\partial \theta}=-\sum_{n=1}^{12}\left(\frac{a}{r}\right)^{n+2} \sum_{m=0}^{n}
\end{aligned}
$$

$$
\begin{aligned}
& \cdot\left(g_{n}^{m} \cdot \cos m \lambda+h_{n}^{m} \cdot \sin m \lambda\right) \cdot \frac{d P_{n}^{m}(\theta)}{d \theta} \\
& -\sum_{n=1}^{5}\left(\frac{r}{a}\right)^{n-1} \sum_{m=0}^{n} \\
& \cdot\left(q_{n}^{m} \cdot \cos m \lambda+s_{n}^{m} \cdot \sin m \lambda\right) \cdot \frac{d P_{n}^{m}(\theta)}{d \theta} \\
B_{\lambda}= & -\frac{1}{r \sin \theta} \frac{\partial V}{\partial \lambda}=\frac{1}{\sin \theta} \sum_{n=1}^{12}\left(\frac{a}{r}\right)^{n+2} \sum_{m=0}^{n} m \\
& \cdot\left(g_{n}^{m} \cdot \sin m \lambda-h_{n}^{m} \cdot \cos m \lambda\right) \cdot P_{n}^{m}(\theta) \\
& +\frac{1}{\sin \theta} \sum_{n=1}^{5}\left(\frac{r}{a}\right)^{n-1} \sum_{m=0}^{n} m \\
& \cdot\left(q_{n}^{m} \cdot \sin m \lambda-s_{n}^{m} \cdot \cos m \lambda\right) \cdot P_{n}^{m}(\theta)
\end{aligned}
$$

and for secular-variation observations are

$$
\begin{aligned}
\dot{B}_{r}= & -\frac{\partial \dot{V}}{\partial r}=\sum_{n=1}^{8}(n+1)\left(\frac{a}{r}\right)^{n+2} \sum_{m=0}^{n} \\
& \cdot\left(\dot{g}_{n}^{m} \cdot \cos m \lambda+\dot{h}_{n}^{m} \cdot \sin m \lambda\right) \cdot P_{n}^{m}(\theta) \\
\dot{B}_{\theta}= & -\frac{1}{r} \frac{\partial \dot{V}}{\partial \theta}=-\sum_{n=1}^{8}\left(\frac{a}{r}\right)^{n+2} \sum_{m=0}^{n} \\
& \cdot\left(\dot{g}_{n}^{m} \cdot \cos m \lambda+\dot{h}_{n}^{m} \cdot \sin m \lambda\right) \cdot \frac{d P_{n}^{m}(\theta)}{d \theta} \\
\dot{B}_{\lambda}= & -\frac{1}{r \sin \theta} \frac{\partial \dot{V}}{\partial \lambda}=\frac{1}{\sin \theta} \sum_{n=1}^{8}\left(\frac{a}{r}\right)^{n+2} \sum_{m=0}^{n} m \\
& \cdot\left(\dot{g}_{n}^{m} \cdot \sin m \lambda-\dot{h}_{n}^{m} \cdot \cos m \lambda\right) \cdot P_{n}^{m}(\theta) .
\end{aligned}
$$

Satellite data are already located in a geocentric coordinate system but surface data are almost invariably located in a geodetic coordinate system, i.e. relative to the mean sea surface of the Earth, which can be approximated by a spheroid. The locations of surface data, and the data themselves, must be transformed from geodetic to geocentric coordinates prior to spherical harmonic modelling.

\section{Data Selection for Secular-Variation Modelling}

Given that secular variation in many parts of the world is quite small the data requirements for secular-variation modelling and prediction, in approximate order of importance, are that

(1) they are based on high-quality absolute observations,

(2) if they are surface data, they have been measured at exactly the same location through time so that they are uncontaminated by crustal fields when differences are taken between data at different times,

(3) the most recent data are available to minimise the errors caused by prediction,

(4) they have been measured over continuous periods of time or near other data measured over continuous periods of time that are long relative to the periods of the disturbance field variations so that these effects can be evaluated and eliminated,

(5) they are evenly distributed over the globe, and 


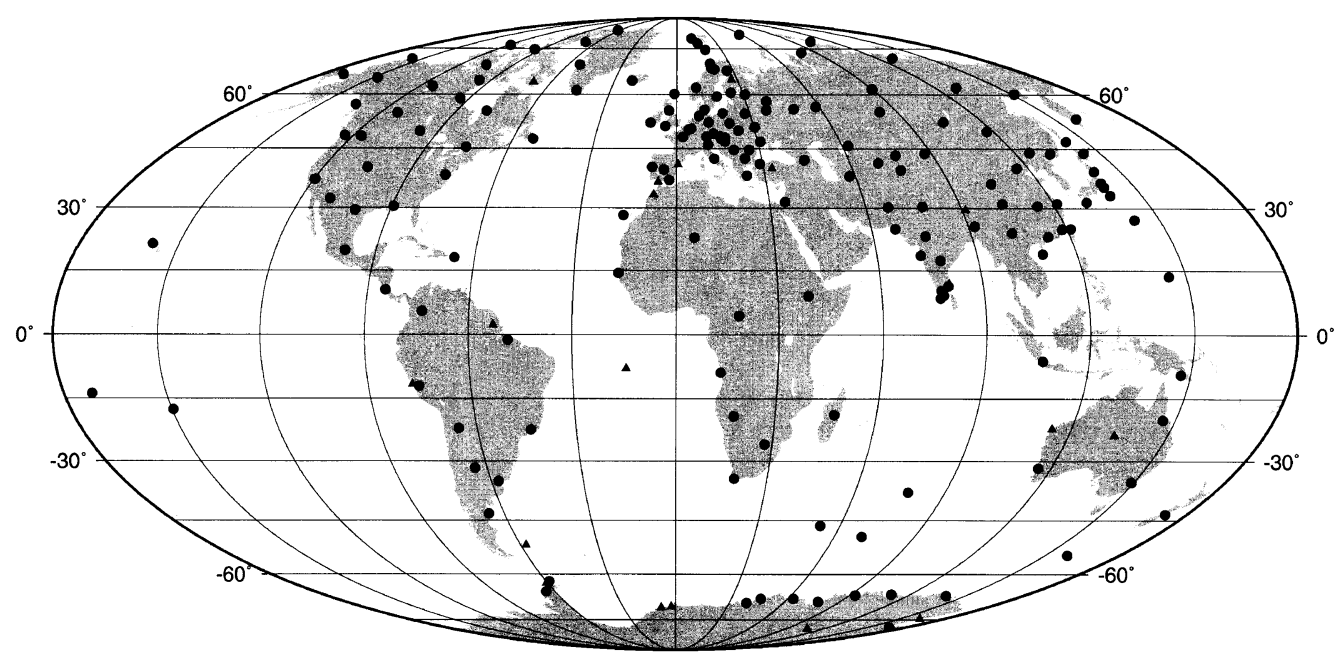

Fig. 1. Spatial distribution of observatories used for secular-variation modelling. The dots show the locations of the 170 observatories where linear prediction filters were applied and the triangles show the locations of 20 other observatories with useful recent secular-variation data.

(6) they have been measured over a long period of time (greater than one year) so that future predictions can be more confidently made based on the historical records.

Data from magnetic observatories satisfy requirements (1), (2), (3), (4) and (6) and, to a lesser extent, so do repeat station data. The observatories and repeat stations, however, do not cover the ocean areas of the Earth and even their distribution on land is far from ideal. To satisfy requirement (5) secular-variation information has been extracted from models based on satellite data. Particular emphasis is placed on requirement (3) for both observatories and repeat stations. The recent conversion of many magnetic observatories from analogue to digital operation, often through the INTERMAGNET programme, has helped considerably in this respect.

A total of 190 observatories was used and their positions are shown in Fig. 1. The disturbance field variations are greatly reduced by using annual means of observatory data. As regards recent data in the final models, 88 observatories had their latest annual means for 1998, 58 for 1997, 8 for 1996 and the remainder for 1995 and before. However for a preliminary model which was put forward as an IGRF candidate model, only 7 observatories had their latest annual means for 1998. The recent temporal distribution of observatory data is shown in Fig. 2. Unfortunately the observatories in the areas of the world where there are few others are, in some cases, experiencing the greatest difficulties in producing timely data. If an observatory has moved for any reason, the data recorded at the original site can often be adjusted to the new site using measured site differences.

Repeat stations were also used for secular-variation modelling. Annual secular-variation estimates were made by taking the difference between the data from two consecutive occupations and dividing by the number of years. Outliers were identified and rejected by comparison of the secularvariation estimates with an existing global model. In an attempt to alleviate the very uneven global distribution, only

\section{Number of observatory secular-variation estimates}

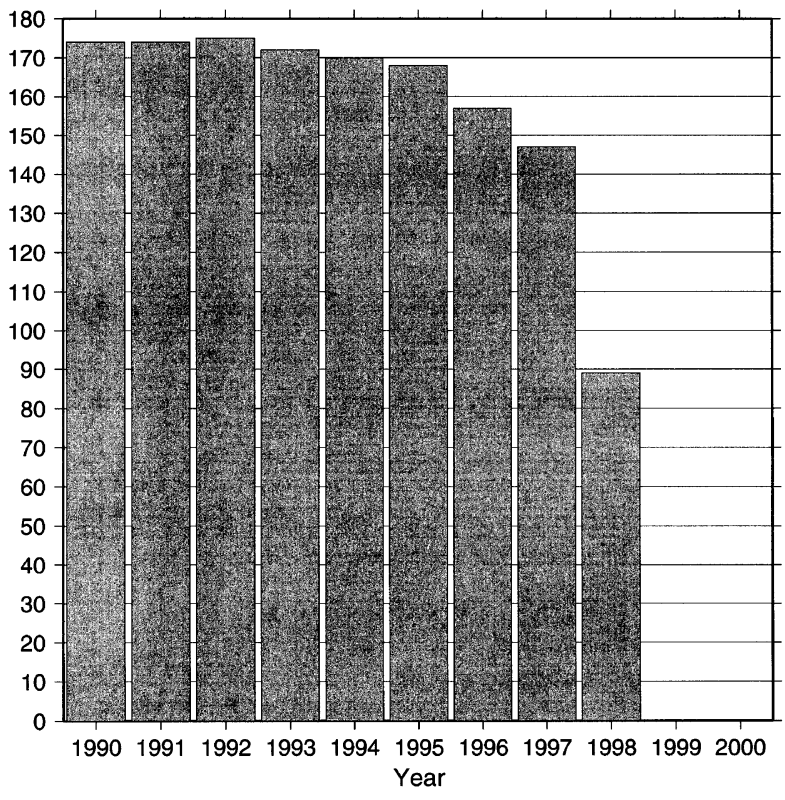

Fig. 2. Temporal distribution of observatory secular-variation data.

three repeat station estimates were selected per equal-area tessera. The tesseral dimensions are fixed in latitude at $5^{\circ}$ but vary in longitude from $5^{\circ}$ at the equator to $120^{\circ}$ near the poles. The distribution of selected repeat stations is shown in Fig. 3.

Equal-area tesserae with dimension $10^{\circ}$ in latitude, which have neither observatory nor repeat-station data, were filled with secular-variation estimates derived from a secularvariation model incorporating satellite data. The data locations are shown in Fig. 4. Details of the models used to compute these synthetic data are given later. 


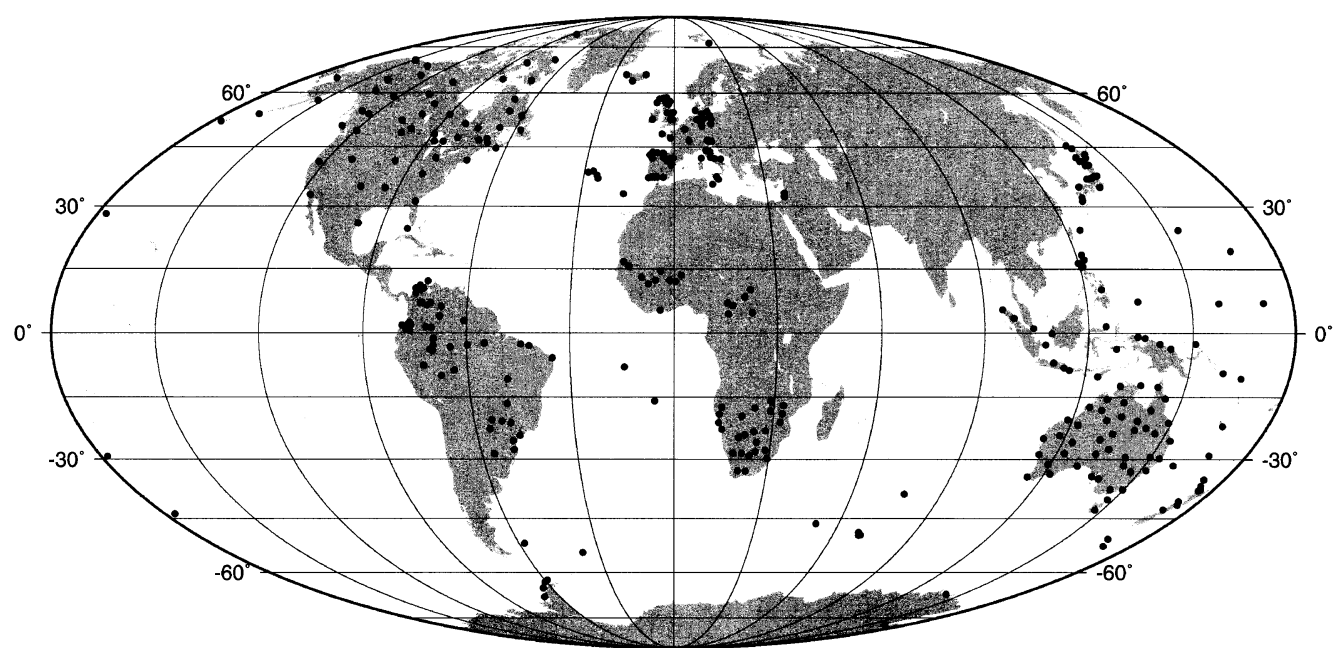

Fig. 3. The 341 selected repeat stations with secular-variation information.

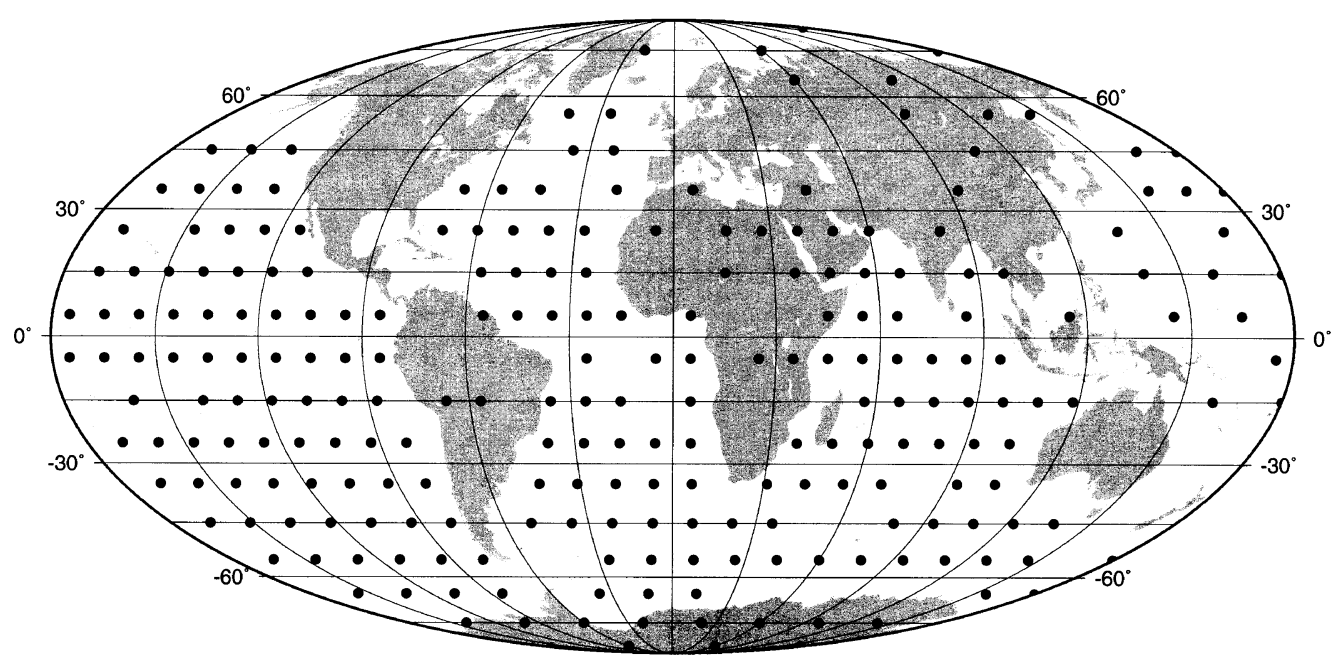

Fig. 4. Locations where synthetic secular-variation values from a 5-year average model were used. This model incorporates data from POGS and Ørsted.

\section{Data Selection for Main-Field Modelling}

For a snapshot model of the present main field the data requirements are slightly different from those for secularvariation modelling. The main emphasis is on the availability of recent data with a good global coverage and with minimal crustal field and disturbance field contamination. The Danish Ørsted satellite data satisfy these requirements.

Ørsted was successfully launched on 23 February 1999 from Vandenburg Air Force Base in California on a Delta II rocket along with the American ARGOS (Advanced Research Global Observation Satellite) and the South African micro-satellite SUNSAT. The satellite has a mass of $62 \mathrm{~kg}$ and measures $34 \times 45 \times 72 \mathrm{~cm}$ when the boom is stowed. The main instruments carried are a Compact Spherical Coil (CSC) fluxgate magnetometer, a variometer measuring three orthogonal components of the magnetic field; an Overhauser magnetometer, an absolute instrument measuring the strength of the magnetic field; and a star imager camera for determining the orientation of the fluxgate magnetometer. These three instruments are mounted at the end of an $8 \mathrm{~m}$ boom to minimise magnetic disturbance from within the satellite body. In the main body there are two other important instruments: a charged particle detector and a Global Positioning System (GPS) receiver. Attitude control is achieved passively by the boom and actively by use of the magnetotorquer coils.

The satellite was launched into a retrograde orbit with the ascending node at the start of the mission being 14:11 local time, apogee $\sim 850 \mathrm{~km}$, perigee $\sim 640 \mathrm{~km}$, inclination $96.5^{\circ}$, nodal period 99.6 minutes, longitude increment $-24.9^{\circ}$ orbit and local time increment -0.88 minutes/day. The satellite velocity is approximately $7.5 \mathrm{~km} / \mathrm{second}$.

Although data recovery from the Overhauser magnetometer has been very high there have been a number of problems obtaining oriented vector data from the CSC fluxgate magnetometer and the star camera. When the boom was deployed on 14 March its orientation relative to the satellite body was about $50^{\circ}$ different from its intended orientation. Over the following two months this decreased to $40^{\circ}$ as the boom ex- 


\section{OERSTED MAGNETIC RESIDUAL CHART}

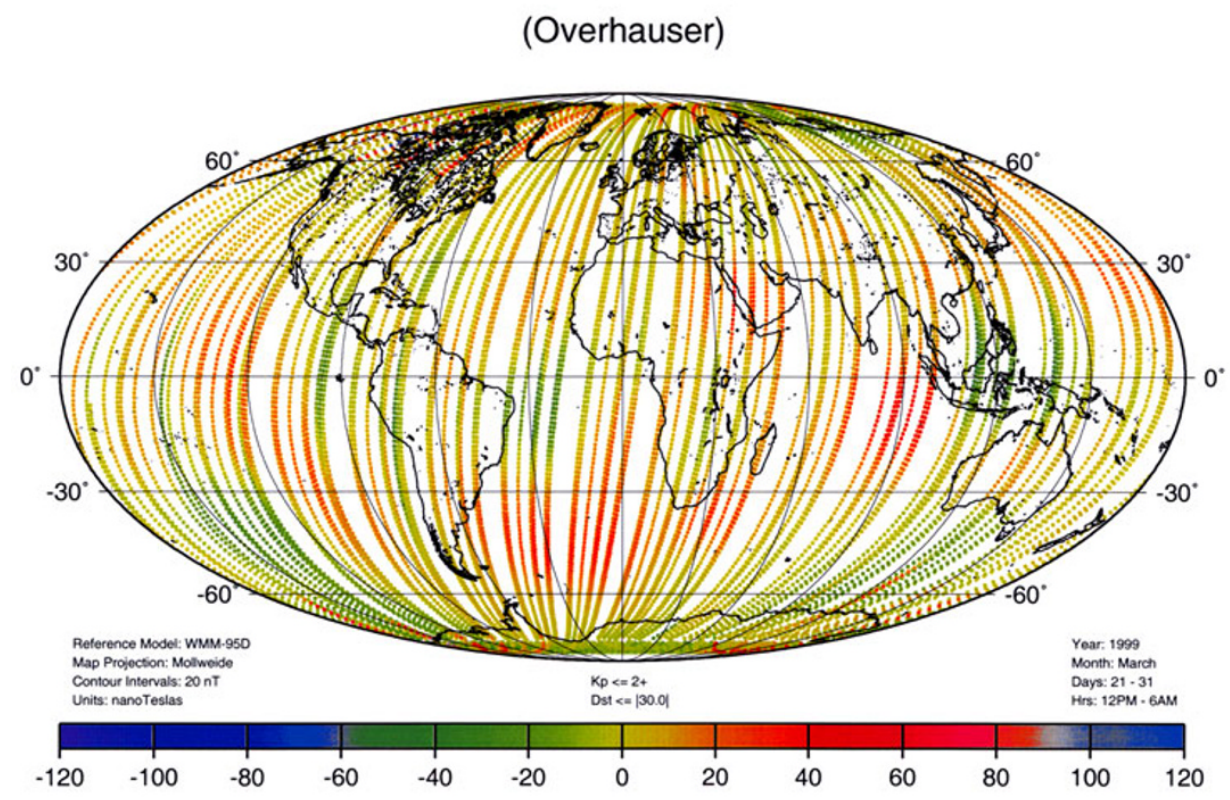

Fig. 5. The differences between the selected Ørsted total intensity observations between 21 and 31 March 1999 and the final revised WMM model as listed in Table 2 .

tended further and tightened the boom release wire. One consequence of this was that the Sun was in the star camera's field of view more often than anticipated, especially in the first few months of the mission, and orientation fixes were unobtainable. Faulty attitude data were therefore sent to the attitude control system and the satellite orbit was destabilised and the star camera was unable to obtain a star fix. The only solution to this negative feedback problem was manual intervention from ground control.

Radiation hits have had quite a significant effect as a result of the mission being close to solar maximum due to the three-year delay in the launch. This particularly affects passes over the so-called South Atlantic anomaly where the Earth's main-field protection given to the satellite from damage due to exposure to charged particles in the radiation belts surrounding the Earth is weakest. The star camera is particularly sensitive to radiation hits and this leads to faulty data being transmitted to the attitude control system. Again, the satellite orbit is destabilised and the star camera is unable to obtain attitude data. By turning down the resolution of the star camera the impact of radiation hits over the South Atlantic anomaly has been reduced, however there has been some loss of accuracy with the attitude data.

Other problems with the vector data include uncertainty with the time stamp associated with the attitude data, thermal boom oscillations resulting in smearing of the images of the stars and a further loss of accuracy with the attitude data, and a $50 \%$ loss of so-called house-keeping data in the first six months of the mission. This includes important data such as temperature which is required for correcting the CSC fluxgate data.

As a result of all these problems and the resulting delays in distributing these vector data, it was decided not to attempt to use them in the World Magnetic Model and to only use the Overhauser pre-calibrated magnetometer data. Since vector data are required in a band straddling the geomagnetic equator in order to eliminate the Backus effect (Stern et al., 1980; Lowes and Martin, 1987), an alternative means of eliminating this effect was employed, which used synthetic vector data from the 1992.5 optimum model derived from Project MAGNET high-level aeromagnetic vector data and Polar Orbiting Geomagnetic Survey (POGS) scalar satellite data during the production of the 1995 model (Quinn et al., 1995). This model was adjusted to Ørsted satellite epochs using secular-variation models.

The crustal field is largely attenuated at the altitude of the satellite and, with the exception of the auroral and polar areas, disturbance field effects at these altitudes are also small. By selecting data during the night-side passes and using global disturbance indices, the disturbance field effects can be further reduced. The time interval selected for modelling extended from 11 March 1999 to 31 May 1999 since this was the only time interval for which preliminary magnetic activity indices were available for data selection. The selected data were divided into eight 10-day or 11-day subsets depending on the number of days per month. Each subset had sufficient data to generate an independent model. An example of the satellite data distribution for one of these subsets is shown in Fig. 5.

\section{Modelling Procedure}

\subsection{Secular-variation prediction}

Prediction of secular variation to 2005.0 was made using linear predictor filters applied to first differences of observatory annual means in the north component $(X)$, the east component $(Y)$ and the vertical component $(Z)$. The historic time series of secular-variation values is characterised in terms of frequencies present by using maximum entropy 


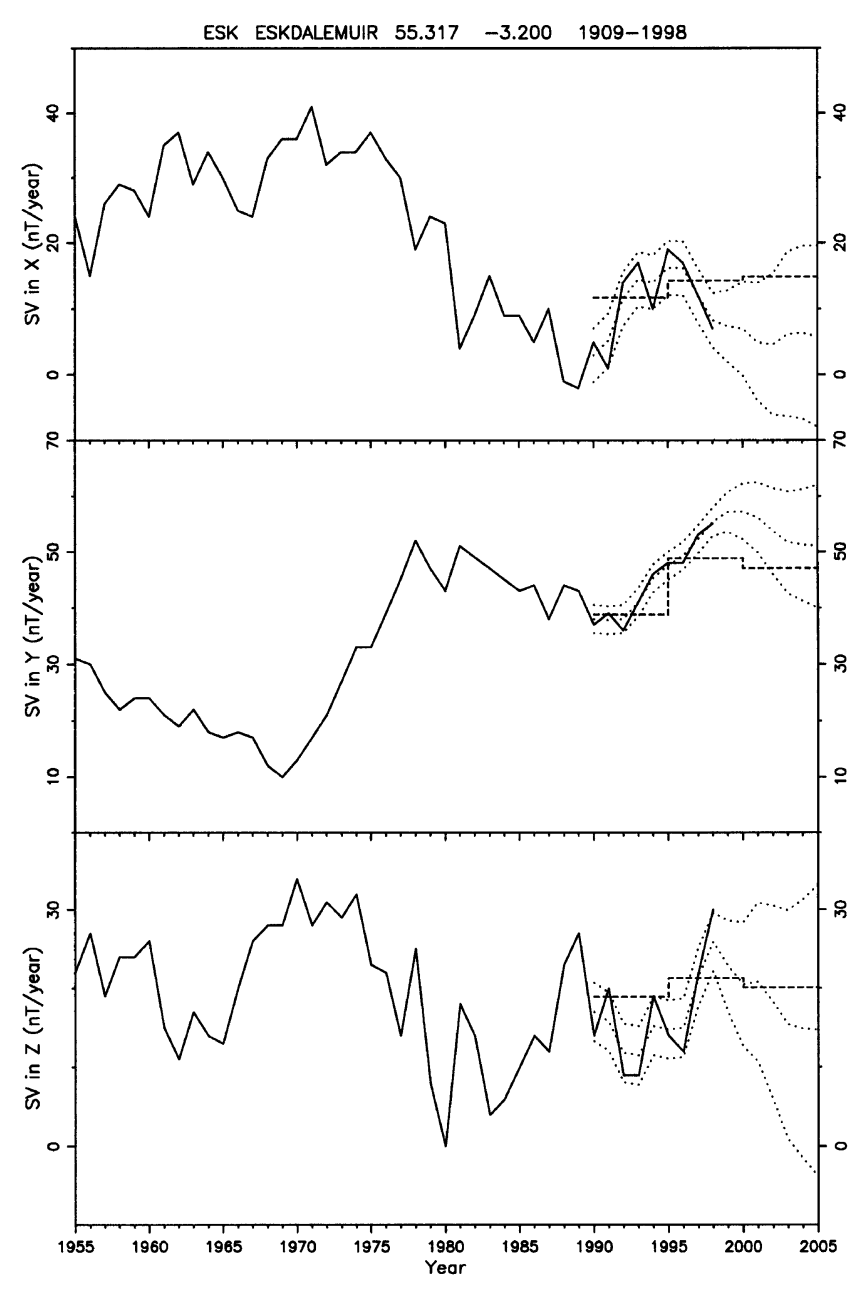

Fig. 6. Observed (solid line), predicted (middle dotted line) and modelled (dashed line) secular variation in $X, Y$ and $Z$ at Eskdalemuir observatory (at $55.317^{\circ} \mathrm{N}, 3.200^{\circ} \mathrm{W}$ ). The annual means from 1909 to 1998 are used for the predictions but only those for 1955 and onwards are shown. The two outer dotted lines are the estimated standard errors.

spectral analysis. The characterisation consists of a finite number of poles that best represents the power spectrum in the $z$-transform plane where $z$ is mapped from complex frequency $f$ by the relation $z \equiv e^{2_{\Pi} i f \Delta}, \Delta$ being the sampling interval in the time domain. The Nyquist interval on the real axis of the $f$-plane maps one-to-one onto the unit circle in the complex $z$-plane. These poles correspond to infinite power spectral density on the unit $z$-circle, i.e. at real frequencies in the Nyquist interval. Such poles provide an accurate representation of the underlying power spectrum, especially if it has sharp, discrete lines. The coefficients of a finite series in the $z$-transform plane which is representative of the power spectrum can be found by comparison of the series with the $z$-transform of the Fourier transform of the autocorrelations in the time domain. These coefficients form the coefficients of a linear filter. The filter is then used to extrapolate the time series beyond its last secular-variation value. Linear prediction is especially successful at extrapolating signals which are smooth and oscillatory, though not necessarily periodic. Any dominant frequencies in the original time series are preserved in the forecast.

As this characterisation requires the secular-variation val-

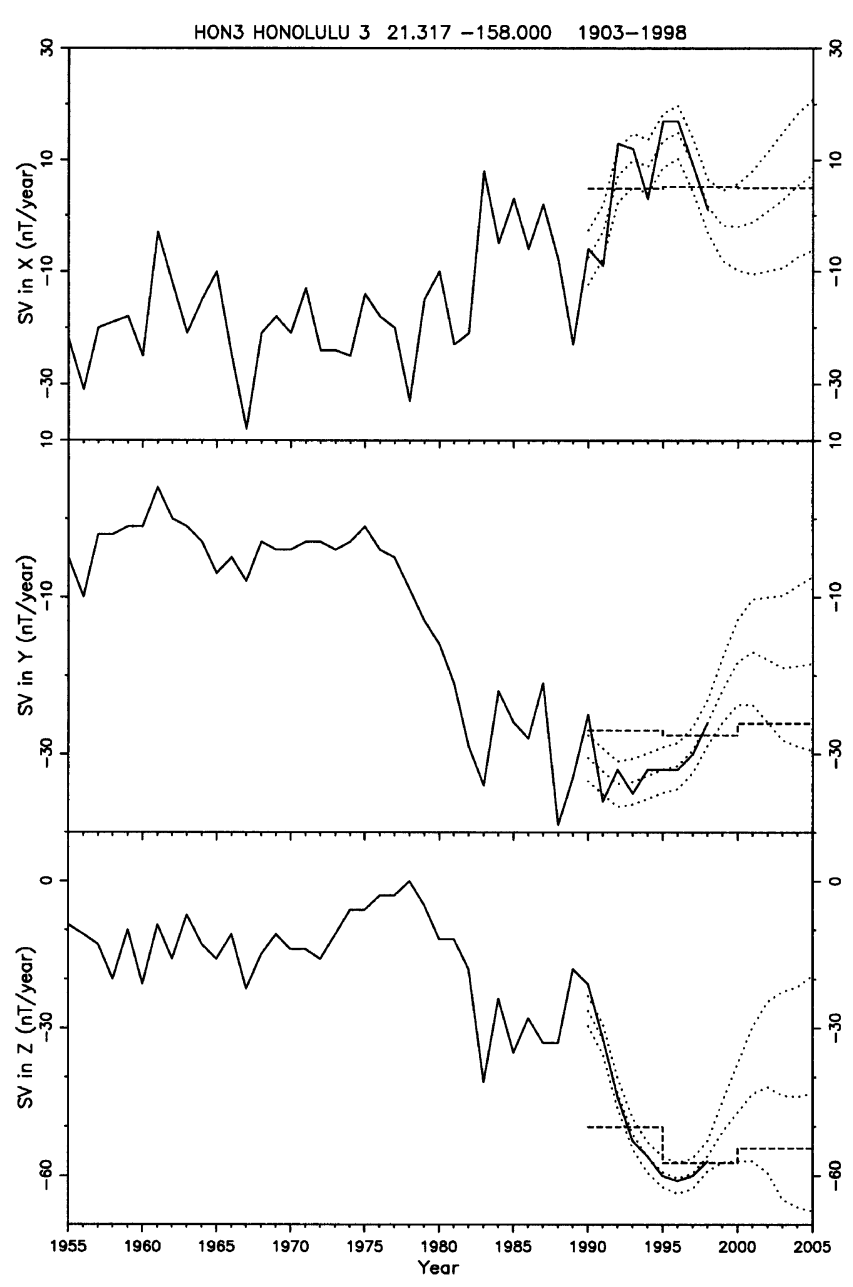

Fig. 7. As Fig. 6 but for Honolulu observatory.

ues to be evenly spaced, any missing values in the secularvariation records were replaced with estimates obtained by linear interpolation if the gap was no more than five years long; otherwise, the earlier part of the record was discarded. In addition, annual means which were not computed from a full twelve months of data were adjusted using the neighbouring annual means so that the resulting secular-variation values were at exactly one year intervals. Some very noisy parts of other records, especially in the vertical component $(Z)$, were also discarded.

The predictions were plotted for each observatory and checked manually to see that they were reasonable. Two examples of these plots are shown in Figs. 6 and 7. In these plots the solid line is the observed secular variation, the middle dotted line is the output from the linear predictor, the two outer dotted lines indicate the estimated 1- $\sigma$ error envelope, and the dashed line connects the values synthesised from the final annual secular-variation spherical harmonic models. As many observatories started in the late 1950 s with the International Geophysical Year in 1957, only the data after 1955 are shown. However, if earlier data exist they were also used to build the linear prediction filters. Along the top of each plot there is the IAGA code, the observatory name, the latitude, the longitude and the time span of data used.

Data from the 20 observatories which had time series too 
Table 1. Statistics of model residuals for Ørsted Overhauser magnetometer data Model.

\begin{tabular}{|c|c|c|c|c|c|c|c|c|c|}
\hline Model & & 1 & 2 & 3 & 4 & 5 & 6 & 7 & 8 \\
\hline & Month & March & March & April & April & April & May & May & May \\
\hline & Day begin & 11 & 21 & 1 & 11 & 21 & 1 & 11 & 21 \\
\hline & Day end & 20 & 31 & 10 & 20 & 30 & 10 & 20 & 31 \\
\hline & Mean day & 77.4 & 84.1 & 96.0 & 105.3 & 114.6 & 126.8 & 135.8 & 144.7 \\
\hline & Records & 9890 & 15115 & 14111 & 13050 & 14664 & 16769 & 14591 & 13926 \\
\hline Iteration & (nT) & & & & & & & & \\
\hline \multirow[t]{2}{*}{1} & Mean & 0.0 & -0.2 & 0.0 & -0.1 & -0.3 & -0.3 & -0.4 & -0.4 \\
\hline & R.m.s. & 12.1 & 11.4 & 11.7 & 11.4 & 12.9 & 12.0 & 10.8 & 11.3 \\
\hline \multirow[t]{2}{*}{2} & Mean & 0.0 & -0.2 & 0.2 & 0.2 & -0.1 & 0.0 & -0.2 & -0.2 \\
\hline & R.m.s. & 10.7 & 9.8 & 10.4 & 10.0 & 11.8 & 10.8 & 9.5 & 9.9 \\
\hline \multirow[t]{2}{*}{3} & Mean & 0.5 & 0.3 & 0.8 & 0.5 & 0.9 & 0.7 & 0.2 & 0.2 \\
\hline & R.m.s. & 10.4 & 9.3 & 10.1 & 9.7 & 11.7 & 10.6 & 9.1 & 9.4 \\
\hline
\end{tabular}

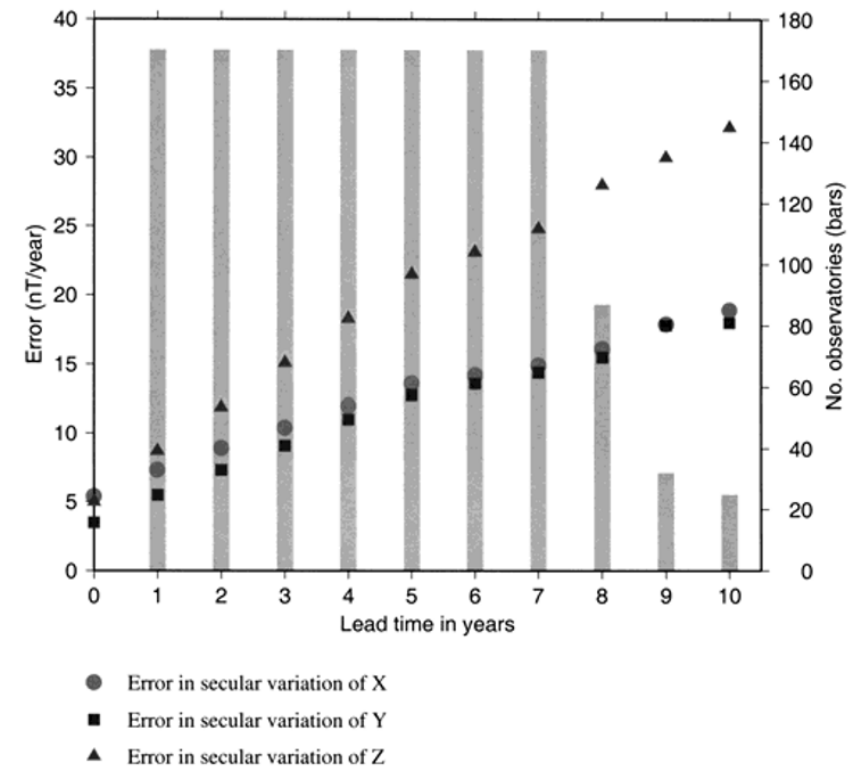

Fig. 8. The global averages of the estimated standard errors assigned to the observatory secular-variation data versus lead time. Those for lead time of 0 years are for observed data and those for lead time $>0$ years are for predicted data. The bars show the number of observatories requiring predictions for the given (maximum) lead times (if the last annual mean for a particular observatory is 1998 , in order to predict to 2005.0 , the maximum lead time is 7 years).

short for the application of linear prediction filters were used by simply computing average secular-variation estimates and assuming that these do not change with time.

Prediction of secular variation at the repeat stations was made either by taking a mean of the secular-variation estimates or, if there were enough recent estimates (more than three since 1988.0), making linear regression fits to the observed average annual secular-variation values at the middates between occupations.

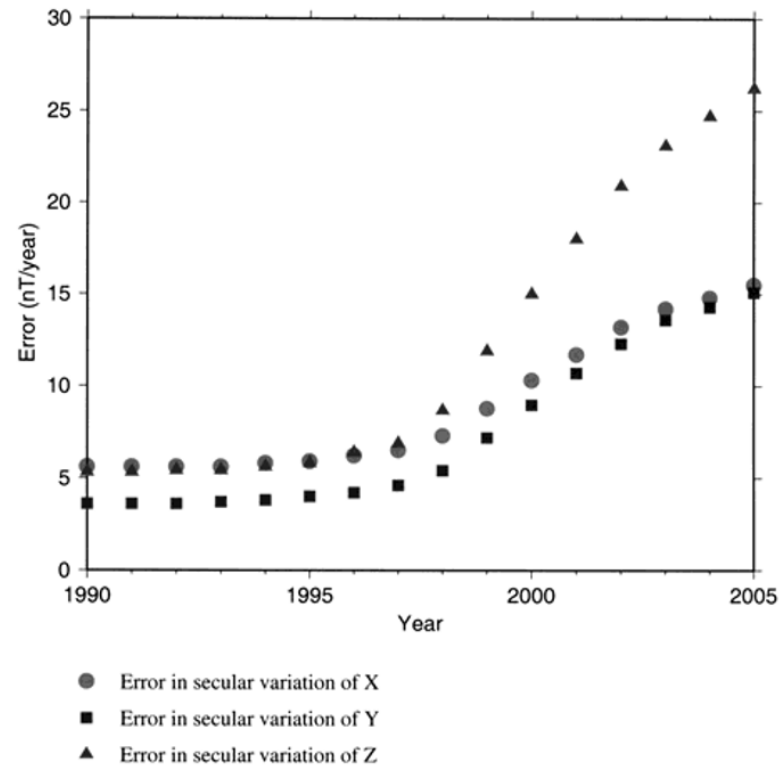

Fig. 9. The global averages of the estimated standard errors assigned to the observatory secular-variation data versus year.

\subsection{Data weighting scheme}

For the main-field models the relative numbers of scalar and vector data in the equatorial region are not equal, but the vector data are more heavily weighted so as to give these synthetic data an overall weight equal to the scalar data contained in a fixed equatorial band about the geomagnetic equator. All data are equal-area weighted using a factor of $\sin \theta$ where $\theta$ is the geocentric colatitude. Since many of the data for the main-field model are total intensity data, and are therefore not linear in the Gauss coefficients, an iterative method is required to determine the coefficients. In the last iteration outliers are down-weighted using the factor

$$
w_{0}=e^{-\left(\frac{\Delta B}{3 \tilde{\sigma}}\right)^{2}}
$$


Table 2. Gauss main-field coefficients for 1995.0 and 2000.0 and secular-variation (SV) coefficients for 2000.0-2005.0.

\begin{tabular}{|c|c|c|c|c|c|c|c|c|c|c|c|}
\hline$g / h$ & $n$ & $m$ & 1995.0 & 2000.0 & SV & $g / h$ & $n$ & $m$ & 1995.0 & 2000.0 & SV \\
\hline$g$ & 1 & 0 & -29686.6 & -29616.0 & 14.7 & $h$ & 6 & 6 & 34.5 & 43.0 & 1.9 \\
\hline$g$ & 1 & 1 & -1781.9 & -1722.7 & 11.1 & $g$ & 7 & 0 & 78.1 & 77.4 & -0.4 \\
\hline$h$ & 1 & 1 & 5311.1 & 5194.5 & -20.4 & $g$ & 7 & 1 & -68.8 & -73.9 & -0.8 \\
\hline$g$ & 2 & 0 & -2196.1 & -2266.7 & -13.6 & $h$ & 7 & 1 & -73.4 & -62.3 & 1.4 \\
\hline$g$ & 2 & 1 & 3071.9 & 3070.2 & -0.7 & $g$ & 7 & 2 & 1.1 & 2.2 & -0.2 \\
\hline$h$ & 2 & 1 & -2370.2 & -2484.8 & -21.5 & $h$ & 7 & 2 & -25.4 & -24.5 & 0.2 \\
\hline$g$ & 2 & 2 & 1684.2 & 1677.6 & -1.8 & $g$ & 7 & 3 & 31.5 & 35.7 & 1.1 \\
\hline$h$ & 2 & 2 & -420.4 & -467.9 & -9.6 & $h$ & 7 & 3 & 3.5 & 8.9 & 0.7 \\
\hline$g$ & 3 & 0 & 1319.3 & 1322.4 & 0.3 & $g$ & 7 & 4 & 3.3 & 7.3 & 0.4 \\
\hline$g$ & 3 & 1 & -2267.9 & -2291.5 & -4.3 & $h$ & 7 & 4 & 20.5 & 23.4 & 0.4 \\
\hline$h$ & 3 & 1 & -254.6 & -224.7 & 6.4 & $g$ & 7 & 5 & 6.8 & 5.2 & 0.0 \\
\hline$g$ & 3 & 2 & 1251.4 & 1255.9 & 0.9 & $h$ & 7 & 5 & 17.4 & 15.0 & -0.3 \\
\hline$h$ & 3 & 2 & 298.3 & 293.0 & -1.3 & $g$ & 7 & 6 & 8.6 & 8.4 & -0.2 \\
\hline$g$ & 3 & 3 & 765.5 & 724.8 & -8.4 & $h$ & 7 & 6 & -24.6 & -27.6 & -0.8 \\
\hline$h$ & 3 & 3 & -418.9 & -486.5 & -13.3 & $g$ & 7 & 7 & -2.3 & -1.5 & -0.2 \\
\hline$g$ & 4 & 0 & 938.2 & 932.1 & -1.6 & $h$ & 7 & 7 & -6.3 & -7.8 & -0.1 \\
\hline$g$ & 4 & 1 & 782.3 & 786.3 & 0.9 & $g$ & 8 & 0 & 24.1 & 23.3 & -0.3 \\
\hline$h$ & 4 & 1 & 260.9 & 273.3 & 2.3 & $g$ & 8 & 1 & 5.2 & 7.3 & 0.6 \\
\hline$g$ & 4 & 2 & 289.9 & 250.6 & -7.6 & $h$ & 8 & 1 & 13.1 & 12.4 & -0.5 \\
\hline$h$ & 4 & 2 & -230.8 & -227.9 & 0.7 & $g$ & 8 & 2 & -3.2 & -8.5 & -0.8 \\
\hline$g$ & 4 & 3 & -415.3 & -401.5 & 2.2 & $h$ & 8 & 2 & -18.9 & -20.8 & 0.1 \\
\hline$h$ & 4 & 3 & 99.5 & 120.9 & 3.7 & $g$ & 8 & 3 & -9.2 & -6.6 & 0.3 \\
\hline$g$ & 4 & 4 & 118.8 & 106.2 & -3.2 & $h$ & 8 & 3 & 6.9 & 8.4 & -0.2 \\
\hline$h$ & 4 & 4 & -302.8 & -302.7 & -0.5 & $g$ & 8 & 4 & -15.4 & -16.9 & -0.2 \\
\hline$g$ & 5 & 0 & -210.6 & -211.9 & -0.9 & $h$ & 8 & 4 & -20.8 & -21.2 & 0.0 \\
\hline$g$ & 5 & 1 & 352.6 & 351.6 & -0.2 & $g$ & 8 & 5 & 4.0 & 8.6 & 0.5 \\
\hline$h$ & 5 & 1 & 42.8 & 42.0 & 0.0 & $h$ & 8 & 5 & 13.0 & 15.5 & 0.1 \\
\hline$g$ & 5 & 2 & 235.5 & 220.8 & -2.5 & $g$ & 8 & 6 & 3.1 & 4.9 & 0.0 \\
\hline$h$ & 5 & 2 & 161.5 & 173.8 & 2.1 & $h$ & 8 & 6 & 8.2 & 9.1 & -0.1 \\
\hline$g$ & 5 & 3 & -123.7 & -134.5 & -2.7 & $g$ & 8 & 7 & -5.0 & -7.8 & -0.6 \\
\hline$h$ & 5 & 3 & -147.4 & -135.0 & 2.3 & $h$ & 8 & 7 & -17.8 & -15.5 & 0.3 \\
\hline$g$ & 5 & 4 & -164.4 & -168.8 & -0.9 & $g$ & 8 & 8 & -8.4 & -7.6 & 0.1 \\
\hline$h$ & 5 & 4 & -56.2 & -38.6 & 3.1 & $h$ & 8 & 8 & -7.3 & -5.4 & 0.2 \\
\hline$g$ & 5 & 5 & -21.9 & -13.3 & 1.7 & $g$ & 9 & 0 & 2.9 & 5.7 & 0.0 \\
\hline$h$ & 5 & 5 & 104.4 & 105.2 & 0.0 & $g$ & 9 & 1 & 7.7 & 8.5 & 0.0 \\
\hline$g$ & 6 & 0 & 69.3 & 73.8 & 1.2 & $h$ & 9 & 1 & -20.3 & -20.4 & 0.0 \\
\hline$g$ & 6 & 1 & 66.3 & 68.2 & 0.2 & $g$ & 9 & 2 & 0.5 & 2.0 & 0.0 \\
\hline$h$ & 6 & 1 & -17.0 & -17.4 & -0.3 & $h$ & 9 & 2 & 14.1 & 13.9 & 0.0 \\
\hline$g$ & 6 & 2 & 66.8 & 74.1 & 1.7 & $g$ & 9 & 3 & -10.2 & -9.8 & 0.0 \\
\hline$h$ & 6 & 2 & 72.3 & 61.2 & -1.7 & $h$ & 9 & 3 & 11.3 & 12.0 & 0.0 \\
\hline$g$ & 6 & 3 & -171.6 & -163.5 & 1.6 & $g$ & 9 & 4 & 9.5 & 7.6 & 0.0 \\
\hline$h$ & 6 & 3 & 68.5 & 63.2 & -0.9 & $h$ & 9 & 4 & -7.2 & -6.2 & 0.0 \\
\hline$g$ & 6 & 4 & -0.3 & -3.8 & -0.1 & $g$ & 9 & 5 & -2.5 & -7.0 & 0.0 \\
\hline$h$ & 6 & 4 & -57.3 & -62.9 & -1.0 & $h$ & 9 & 5 & -7.2 & -8.6 & 0.0 \\
\hline$g$ & 6 & 5 & 17.3 & 17.1 & -0.3 & $g$ & 9 & 6 & -2.3 & -2.0 & 0.0 \\
\hline$h$ & 6 & 5 & 1.6 & 0.2 & -0.1 & $h$ & 9 & 6 & 9.2 & 9.4 & 0.0 \\
\hline$g$ & 6 & 6 & -89.2 & -85.1 & 0.8 & $g$ & 9 & 7 & 6.9 & 9.2 & 0.0 \\
\hline
\end{tabular}


Table 2. (continued).

\begin{tabular}{|c|c|c|c|c|c|}
\hline$g / h$ & $n$ & $m$ & 1995.0 & 2000.0 & SV \\
\hline$h$ & 9 & 7 & 7.6 & 5.0 & 0.0 \\
\hline$g$ & 9 & 8 & -0.4 & -2.2 & 0.0 \\
\hline$h$ & 9 & 8 & -8.1 & -8.4 & 0.0 \\
\hline$g$ & 9 & 9 & -6.5 & -6.6 & 0.0 \\
\hline$h$ & 9 & 9 & 2.7 & 3.2 & 0.0 \\
\hline$g$ & 10 & 0 & -3.0 & -2.2 & 0.0 \\
\hline$g$ & 10 & 1 & -3.5 & -5.7 & 0.0 \\
\hline$h$ & 10 & 1 & 3.1 & 0.9 & 0.0 \\
\hline$g$ & 10 & 2 & 2.9 & 1.6 & 0.0 \\
\hline$h$ & 10 & 2 & 1.3 & -0.7 & 0.0 \\
\hline$g$ & 10 & 3 & -4.3 & -3.7 & 0.0 \\
\hline$h$ & 10 & 3 & 2.9 & 3.9 & 0.0 \\
\hline$g$ & 10 & 4 & -3.0 & -0.6 & 0.0 \\
\hline$h$ & 10 & 4 & 5.6 & 4.8 & 0.0 \\
\hline$g$ & 10 & 5 & 2.7 & 4.1 & 0.0 \\
\hline$h$ & 10 & 5 & -3.5 & -5.3 & 0.0 \\
\hline$g$ & 10 & 6 & 2.9 & 2.2 & 0.0 \\
\hline$h$ & 10 & 6 & -0.6 & -1.0 & 0.0 \\
\hline$g$ & 10 & 7 & 1.0 & 2.2 & 0.0 \\
\hline$h$ & 10 & 7 & -2.6 & -2.4 & 0.0 \\
\hline$g$ & 10 & 8 & 4.0 & 4.6 & 0.0 \\
\hline$h$ & 10 & 8 & 2.4 & 1.3 & 0.0 \\
\hline$g$ & 10 & 9 & 3.6 & 2.3 & 0.0 \\
\hline$h$ & 10 & 9 & -1.6 & -2.3 & 0.0 \\
\hline$g$ & 10 & 10 & 0.6 & 0.1 & 0.0 \\
\hline$h$ & 10 & 10 & -6.6 & -6.4 & 0.0 \\
\hline$g$ & 11 & 0 & 1.8 & 3.3 & 0.0 \\
\hline$g$ & 11 & 1 & -1.4 & -1.1 & 0.0 \\
\hline$h$ & 11 & 1 & -0.1 & -1.5 & 0.0 \\
\hline$g$ & 11 & 2 & -3.4 & -2.4 & 0.0 \\
\hline$h$ & 11 & 2 & 1.1 & 0.7 & 0.0 \\
\hline$g$ & 11 & 3 & 1.3 & 2.6 & 0.0 \\
\hline$h$ & 11 & 3 & -3.3 & -1.1 & 0.0 \\
\hline$g$ & 11 & 4 & -0.7 & -1.3 & 0.0 \\
\hline$h$ & 11 & 4 & -1.6 & -2.3 & 0.0 \\
\hline$g$ & 11 & 5 & -0.2 & -1.7 & 0.0 \\
\hline$h$ & 11 & 5 & 1.6 & 1.3 & 0.0 \\
\hline
\end{tabular}

where $\Delta B$ is the residual of the observation over the model from the second iteration and $\bar{\sigma}$ is the global root mean square (r.m.s.) error of the model from the second iteration. By the third and last iteration, the input model is presumed to be close to the final model, so the net effect of this weight factor is to down-weight outliers, particularly those found in the auroral zones, which are most likely associated with fieldaligned currents and the auroral electrojets, which are always present. Satellite orbits must penetrate the field-aligned currents in the polar regions. At such times the magnetic field is

\begin{tabular}{crrrrr}
\hline$g / h$ & $n$ & $m$ & 1995.0 & 2000.0 & $\mathrm{SV}$ \\
\hline$g$ & 11 & 6 & -0.7 & -0.6 & 0.0 \\
$h$ & 11 & 6 & 0.1 & -0.6 & 0.0 \\
$g$ & 11 & 7 & -0.7 & 0.4 & 0.0 \\
$h$ & 11 & 7 & -1.4 & -2.8 & 0.0 \\
$g$ & 11 & 8 & 1.4 & 0.7 & 0.0 \\
$h$ & 11 & 8 & -2.3 & -1.6 & 0.0 \\
$g$ & 11 & 9 & -0.3 & -0.3 & 0.0 \\
$h$ & 11 & 9 & -0.7 & -0.1 & 0.0 \\
$g$ & 11 & 10 & 2.1 & 2.3 & 0.0 \\
$h$ & 11 & 10 & -2.1 & -1.9 & 0.0 \\
$g$ & 11 & 11 & 4.1 & 4.2 & 0.0 \\
$h$ & 11 & 11 & 1.4 & 1.4 & 0.0 \\
$g$ & 12 & 0 & -1.8 & -1.5 & 0.0 \\
$g$ & 12 & 1 & 0.4 & -0.2 & 0.0 \\
$h$ & 12 & 1 & 0.3 & -1.0 & 0.0 \\
$g$ & 12 & 2 & 0.0 & -0.3 & 0.0 \\
$h$ & 12 & 2 & 1.2 & 0.7 & 0.0 \\
$g$ & 12 & 3 & -0.4 & 0.5 & 0.0 \\
$h$ & 12 & 3 & 1.1 & 2.2 & 0.0 \\
$g$ & 12 & 4 & 0.7 & 0.2 & 0.0 \\
$h$ & 12 & 4 & -2.9 & -2.5 & 0.0 \\
$g$ & 12 & 5 & 0.4 & 0.9 & 0.0 \\
$h$ & 12 & 5 & 0.3 & -0.2 & 0.0 \\
$g$ & 12 & 6 & 0.5 & -1.4 & 0.0 \\
$h$ & 12 & 6 & 0.5 & 0.0 & 0.0 \\
$g$ & 12 & 7 & 0.5 & 0.6 & 0.0 \\
$h$ & 12 & 7 & -0.8 & -0.2 & 0.0 \\
$g$ & 12 & 8 & -0.5 & -0.6 & 0.0 \\
$h$ & 12 & 8 & 0.7 & 0.0 & 0.0 \\
$g$ & 12 & 9 & 0.4 & -1.0 & 0.0 \\
$h$ & 12 & 9 & 0.2 & 0.2 & 0.0 \\
$g$ & 12 & 10 & 0.2 & -0.3 & 0.0 \\
$h$ & 12 & 10 & -1.4 & -0.9 & 0.0 \\
$g$ & 12 & 11 & 0.4 & 0.3 & 0.0 \\
$h$ & 12 & 11 & -0.4 & -0.2 & 0.0 \\
$g$ & 12 & 12 & 0.4 & 0.4 & 0.0 \\
$h$ & 12 & 12 & 0.9 & 1.0 & 0.0 \\
\hline & & & & &
\end{tabular}

not curl-free, and so does not admit a scalar potential satisfying Laplace's equation. This weight factor provides some control over this situation and is used in lieu of discarding outliers, which is often done.

For the secular-variation models error assignment is based on actual past prediction errors (up to a maximum lead time of 10 years) both globally and locally. For the observed values the errors are based on the r.m.s. residuals to quadratic fits made to data over a five-year period. The global mean 1- $\sigma$ errors for each component are shown in Fig. 8-the values 


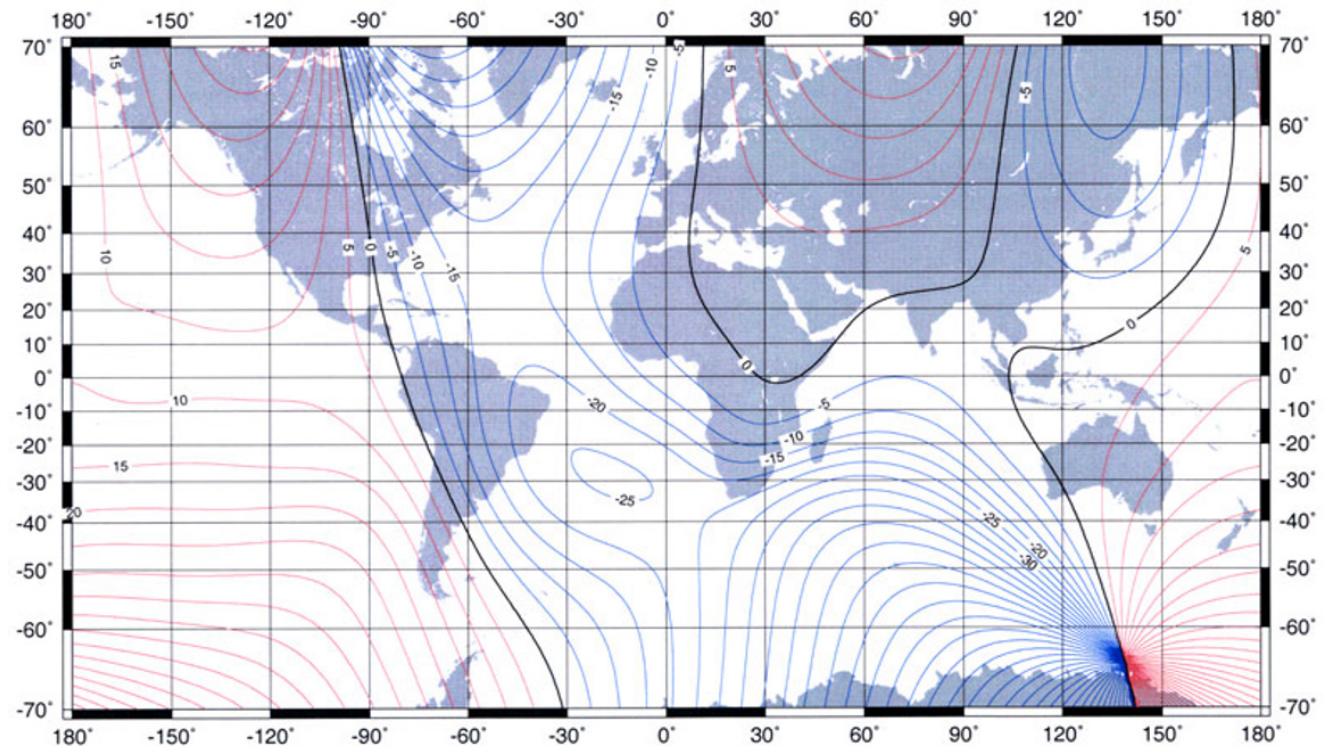

Fig. 10. Declination (magnetic variation) at 2000.0 from the World Magnetic Model(WMM2000). Red—positive (east), blue-negative (west), black—zero (agonic line). Contour interval is $5^{\circ}$ and the projection is Mercator. This is an example of an isogonic chart.

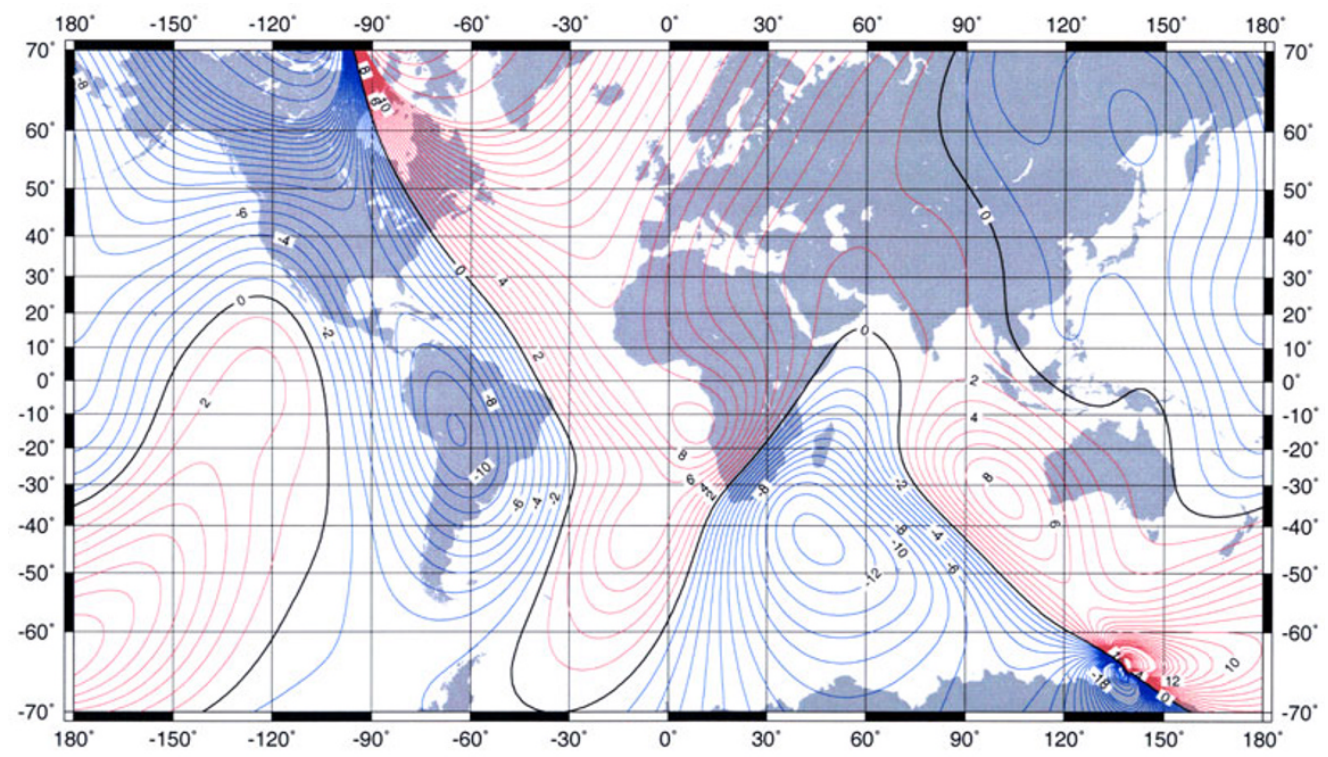

Fig. 11. Annual rate of change of declination for 2000.0 to 2005.0 from the World Magnetic Model (WMM2000). Red-easterly change, blue-westerly change, black-zero change. Contour interval is $1^{\prime} /$ year up to $\pm 20^{\prime} /$ year, thereafter $5^{\prime} /$ year, and the projection is Mercator. This is an example of an isoporic chart.

for lead time of 0 years are the means of the errors for the observed values. It can be seen that the errors for predicting secular variation in $Z$ are higher than for $X$ and $Y$. Also shown in Fig. 8 is the number of observatories requiring predictions for each lead-time. As most observatories had annual mean values for 1998 the most common maximum lead-time is 7 years and this was for predictions for 2005.0.

Shown in Fig. 9 is the mean error of the data (observed and predicted) by year. The diagonal terms of the weight matrix in the least-squares analysis for determining the spherical harmonic coefficients are $1 / \sigma^{2}$, while the off-diagonal terms were set to zero, i.e. secular-variation data were assumed to be uncorrelated.

For the secular-variation estimates derived from repeat stations the errors were set at five times greater than the mean observatory errors as shown in Fig. 8. The errors are increased by a further $50 \%$ if one or both observations making up a secular-variation estimate had not been reduced to a magnetically quiet level.

The satellite-based secular-variation values were assigned errors $70 \%$ greater than the mean errors of the observatory estimates. 
Table 3. Statistics of WMM2000 residuals for observatory annual means and repeat station data.

\begin{tabular}{lrrrr}
\hline Observatories & 1995 & 1996 & 1997 & 1998 \\
\hline Mean $X$ residual (nT) & -26 & -23 & -27 & -50 \\
R.m.s. $X$ residual & 221 & 194 & 199 & 223 \\
Mean $Y$ residual & -16 & -20 & -19 & -33 \\
R.m.s. $Y$ residual & 219 & 224 & 228 & 250 \\
Mean $Z$ residual & 12 & 9 & 10 & -11 \\
R.m.s. $Z$ residual & 231 & 217 & 228 & 240 \\
Mean $F$ residual & -16 & -16 & -19 & -30 \\
R.m.s. $F$ residual & 249 & 225 & 222 & 239 \\
Number of usable data locations & 170 & 154 & 151 & 99 \\
\hline Repeat stations & & & & \\
\hline Mean $X$ residual & -14 & -1 & -1 & -12 \\
R.m.s. $X$ residual & 120 & 152 & 189 & 222 \\
Mean $Y$ residual & 6 & -2 & 20 & 0 \\
R.m.s. $Y$ residual & 103 & 145 & 138 & 163 \\
Mean $Z$ residual & 0 & -39 & -78 & 2 \\
R.m.s. $Z$ residual & 159 & 219 & 186 & 221 \\
Mean $F$ residual & -6 & -37 & 13 & 5 \\
R.m.s. $F$ residual & 143 & 205 & 183 & 219 \\
Number of usable data locations & 481 & 189 & 101 & 123 \\
\hline
\end{tabular}

\subsection{Derivation of WMM2000}

As the deadline for the submission of IGRF candidate models was early in 1999 a very preliminary WMM2000 was derived at this stage to provide a UK/US candidate model. Sixteen annual secular-variation models were computed, each valid from the mid-point of one year to the midpoint of the next year, for the period 1990 to 2005. In these models values at the locations shown in Fig. 4 were computed from a secular-variation model which included secularvariation information derived from POGS data (spanning 2.7 years) and recent observatory and repeat station data. The three five-year average models were taken as weighted mean values of the annual coefficients. For example, for the secular-variation model for 2000.0-2005.0, a mean of the coefficients of the models for 2000.0, 2001.0, 2002.0, 2003.0, 2004.0 and 2005.0 was used with half weight being given to the 2000.0 and 2005.0 coefficients and unit weight to the others. For the IGRF 2000 candidate model, the base epoch model of WMM-95 at 1992.5 (Quinn et al., 1995), derived from POGS and Project MAGNET data, was updated to 2000.0 using the secular-variation models for 1990.0-1995.0 and 1995.0-2000.0. This model, truncated at degree 10, and the predictive secular-variation model for 2000.0-2005.0, was the UK/US candidate IGRF 2000 model.

An iterative procedure was adopted for the derivation of the final WMM2000. The first step was the derivation of the secular-variation models using the same method as above but incorporating new observatory and repeat station data received since the IGRF 2000 candidate model had been computed.

The next step was the computation of an initial main-field model for 2000.0 which incorporated Ørsted data. This step in itself was an iterative process as the bulk of the data for the main-field model was total intensity data and, being nonlinear in the Gauss coefficients, an a priori or initial-guess model was required. In addition it was suspected that there were errors in the model used to generate the equatorial vector data but, by strengthening the influence of the Ørsted scalar data on these synthetic vector data at each iteration, it was hoped to reduce them.

Eight main-field models were generated, each based on data from 10 or 11 consecutive days, depending on the number of days in each month, from 11 March to 31 May 1999. Preliminary models with synthetic vector data within $20^{\circ}$ of the geomagnetic equator were synthesised from the 1992.5 optimum model (Quinn et al., 1995) updated to the base epoch of the selected Ørsted data using the initial secular-variation models for 1990.0-1995.0 and 1995.0-2000.0. The 1992.5 optimum model updated to 1995.0 is the preliminary revised WMM-95 model. Every 20th Ørsted data sample was selected resulting in a 20 -second data series. This was further decimated by only selecting data from periods when local time was between midnight and 2 am, $K p \leq 2_{+}$and Dst $\leq|30 \mathrm{nT}|$. The a priori model was the same model that was used to generate the equatorial vector data. For the next iteration the equatorial vector data were computed from the model from the previous iteration and combined with the scalar data. For each of the eight models there were three iterations. The eight models obtained from the third iteration were adjusted to 2000.0, using eight degree-12 secular-variation models derived by differencing the preliminary revised WMM-95 model with each of the eight Ørsted 
Table 4. Continental US 2000.0 Schmidt-normalized Gauss coefficients. Latitude limits $20^{\circ} \mathrm{N}$ to $54^{\circ} \mathrm{N}$, longitude limits $229^{\circ} \mathrm{E}$ to $299^{\circ} \mathrm{E}$.

\begin{tabular}{|c|c|c|c|c|c|}
\hline$n$ & $m$ & $g_{n}^{m}(\mathrm{nT})$ & $h_{n}^{m}(\mathrm{nT})$ & $\dot{g}_{n}^{m}(\mathrm{nT} / \mathrm{yr})$ & $\dot{h}_{n}^{m}(\mathrm{nT} / \mathrm{yr})$ \\
\hline 1 & 0 & -29193.1 & & -58.5 & \\
\hline 1 & 1 & 2058.2 & 2165.7 & -120.2 & -110.7 \\
\hline 2 & 0 & 3383.8 & & 186.7 & \\
\hline 2 & 1 & -4202.2 & 5593.2 & 176.4 & -20.0 \\
\hline 2 & 2 & 6363.0 & 8486.1 & 167.5 & -327.3 \\
\hline 3 & 0 & -10439.3 & & -170.2 & \\
\hline 3 & 1 & -2218.6 & 601.8 & -27.2 & 177.7 \\
\hline 3 & 2 & -11006.3 & -18175.1 & -113.6 & 368.0 \\
\hline 3 & 3 & -5471.0 & 2808.0 & 346.3 & 99.5 \\
\hline 4 & 0 & 7262.2 & & 54.5 & \\
\hline 4 & 1 & 8582.8 & -8404.0 & -90.8 & -155.2 \\
\hline 4 & 2 & 3127.8 & 9466.1 & -13.9 & -127.8 \\
\hline 4 & 3 & 13281.8 & -10850.7 & -304.6 & -61.1 \\
\hline 4 & 4 & 680.5 & -1720.1 & -29.6 & 232.5 \\
\hline 5 & 0 & -1359.2 & & -1.3 & \\
\hline 5 & 1 & -4176.9 & 3388.1 & 53.6 & 66.6 \\
\hline 5 & 2 & 4562.8 & 1467.7 & 16.0 & -20.0 \\
\hline 5 & 3 & -7506.5 & 4643.6 & 89.8 & 8.6 \\
\hline 5 & 4 & 3198.7 & 3898.4 & 8.8 & -126.4 \\
\hline 5 & 5 & 89.0 & 963.1 & -88.9 & 7.6 \\
\hline 6 & 0 & 577.4 & & -4.0 & \\
\hline 6 & 1 & 487.5 & -58.8 & -6.7 & -12.5 \\
\hline 6 & 2 & -1374.2 & -1256.5 & -5.6 & 15.5 \\
\hline 6 & 3 & 690.5 & 197.6 & -2.6 & -1.9 \\
\hline 6 & 4 & -1261.9 & -1421.0 & 1.3 & 20.9 \\
\hline 6 & 5 & -440.8 & 274.6 & 25.4 & -2.0 \\
\hline 6 & 6 & -161.4 & 45.4 & -3.6 & -15.5 \\
\hline
\end{tabular}

main-field models over the appropriate time interval. One model was then calculated by taking the weighted mean of the eight models where the weights were the inverses of the r.m.s. fits of each model to the input data. These r.m.s. fits were of the order of $11 \mathrm{nT}$.

The next step was the computation of better secularvariation models using better synthetic data in the areas devoid of observatory and repeat station data, and some new observatory data. The synthetic data were computed from a model of secular variation derived from the difference between the preliminary revised WMM-95 model at 1995.0 and the Ørsted-based model at 2000.0 just derived. As the differences between these secular-variation models and the previous models were predominantly in the areas where only the synthetic data were used, it is hoped that any improvements to these synthetic data by using more recent and absolute satellite data are passed on to the final secular-variation models. New predictions of secular variation at the observatories where new data had been received were made and the secular-variation errors were recomputed. Again, three five-year average secular-variation models were derived.

The final step is the recomputation of the main-field model for 2000.0 using the same method described above, but with revised secular-variation models for the two 5-year time intervals between 1990 and 2000 for reduction of satellite data to epoch, plus a revised predictive secular-variation model for the 5-year interval 2000.0-2005.0. This time, synthetic vector data only within $15^{\circ}$ of the geomagnetic equator were included. Both controlled the Backus effect equally well. Models with the larger band about the geomagnetic equator were also generated but were statistically indistinguishable from the narrow-band models. The models with the least amount of synthetic data were considered the most desirable. So the narrow-band model was chosen for the final model. The 1995.0 model was also recomputed using the new secular-variation models and this is called the final revised WMM-95 model.

The fit of the model to the Ørsted data for each of the eight models generated in the final computation is given in Table 1 . Included are the mean epoch of each model, the number of days used in each model, and the number of Ørsted scalar magnetic field observations (records) used in each model. These statistics are computed with respect to data without considering the weight factor $w_{o}$ discussed in the previous 
Table 5. Alaska 2000.0 Schmidt-normalized Gauss coefficients. Latitude limits $42^{\circ} \mathrm{N}$ to $74^{\circ} \mathrm{N}$, longitude limits $160^{\circ} \mathrm{E}$ to $236^{\circ} \mathrm{E}$.

\begin{tabular}{rrrrrr}
\hline$n$ & $m$ & $g_{n}^{m}(\mathrm{nT})$ & \multicolumn{1}{c}{$h_{n}^{m}(\mathrm{nT})$} & $\dot{g}_{n}^{m}(\mathrm{nT} / \mathrm{yr})$ & $\dot{h}_{n}^{m}(\mathrm{nT} / \mathrm{yr})$ \\
\hline 1 & 0 & -34679.4 & & 1.6 & \\
1 & 1 & -4184.9 & 3966.0 & -2.3 & -47.1 \\
2 & 0 & 11276.3 & & 53.4 & \\
2 & 1 & 1019.9 & -7026.7 & 24.4 & 78.9 \\
2 & 2 & -1133.4 & -10463.8 & 14.2 & 21.5 \\
3 & 0 & -10123.4 & & -116.5 & \\
3 & 1 & 9395.9 & 12116.4 & -7.3 & -126.2 \\
3 & 2 & 5769.4 & 8498.3 & -43.4 & 0.9 \\
3 & 3 & 3630.5 & -7354.4 & -9.1 & 13.2 \\
4 & 0 & 2280.6 & & 85.5 & \\
4 & 1 & -10129.1 & -8789.7 & -18.0 & 76.7 \\
4 & 2 & -2539.8 & 432.5 & 43.4 & -17.3 \\
4 & 3 & -2767.9 & 5813.1 & -14.2 & 10.2 \\
4 & 4 & 804.9 & -1616.7 & -14.2 & 30.6 \\
5 & 0 & 1406.7 & & -24.4 & -13.9 \\
5 & 1 & 3408.0 & 1832.6 & 9.3 & 6.8 \\
5 & 2 & 918.3 & -1651.4 & -22.1 & -3.7 \\
5 & 3 & 218.6 & -1202.0 & 11.3 & -2.2 \\
5 & 4 & -610.5 & 493.0 & -7.3 & \\
5 & 5 & 204.2 & -100.2 & & -1 \\
\hline
\end{tabular}

Table 6. Hawaii 2000.0 Schmidt-normalized Gauss coefficients. Latitude limits $17^{\circ} \mathrm{N}$ to $24^{\circ} \mathrm{N}$, longitude limits $198^{\circ} \mathrm{E}$ to $207^{\circ} \mathrm{E}$.

\begin{tabular}{rrrrrr}
\hline$n$ & $m$ & $g_{n}^{m}(\mathrm{nT})$ & $h_{n}^{m}(\mathrm{nT})$ & $\dot{g}_{n}^{m}(\mathrm{nT} / \mathrm{yr})$ & $\dot{h}_{n}^{m}(\mathrm{nT} / \mathrm{yr})$ \\
\hline 1 & 0 & -25078.1 & & -2.2 & \\
1 & 1 & 2179.2 & 3150.1 & 3.9 & 6.4 \\
2 & 0 & -7414.8 & & 24.5 & \\
2 & 1 & 7740.0 & -1557.2 & -17.0 & -14.9 \\
2 & 2 & 7924.6 & -1609.5 & -16.7 & 35.5 \\
3 & 0 & -328.3 & & -17.1 & \\
3 & 1 & -5868.8 & 583.0 & 22.6 & -11.2 \\
3 & 2 & 1864.0 & 436.4 & -10.0 & -13.7 \\
3 & 3 & 3247.8 & 204.8 & -15.1 & -2.7 \\
\hline
\end{tabular}

Section. Thus the statistics tend to be artificially higher than would be the case had we included the weight factor, or had we chosen to discard data as is often done. An example of the residuals for one 10-day Ørsted dataset used for modelling is presented in Fig. 5. The residuals are computed at the Orsted data epoch with respect to the internal degree 12 model only. The residuals include both ionospheric and lithospheric noise. The statistics in Table 1 include the effect of both the internal degree 12 model and the external degree 5 model.

\section{Modelling Results}

The coefficients of the final main-field models for 1995.0 and 2000.0, and the final secular-variation model for 2000.0-
2005.0 are listed in Table 2.

Shown in Figs. 10 and 11 are Mercator maps of declination at 2000.0 and its annual rate of change for the period 2000.0 to 2005.0, both computed using WMM2000.

The geomagnetic poles, otherwise known as the dipole poles, can be computed from the first three Gauss coefficients. From the WMM2000 coefficients for 2000.0 the geomagnetic north pole is at longitude $71.65^{\circ} \mathrm{W}$ and geodetic latitude $79.60^{\circ} \mathrm{N}$ and the geomagnetic south pole is at longitude $108.35^{\circ} \mathrm{E}$ and geodetic latitude $79.60^{\circ} \mathrm{S}$.

The magnetic poles, otherwise known as the dip poles, are computed from all the Gauss coefficients using an iterative method. At 2000.0 the north magnetic pole is located at longitude $109.37^{\circ} \mathrm{W}$ and geodetic latitude $80.81^{\circ} \mathrm{N}$ and the 
south magnetic pole is at longitude $138.31^{\circ} \mathrm{E}$ and geodetic latitude $64.67^{\circ} \mathrm{S}$. In practice the geomagnetic field is not exactly vertical at these dip poles but on oval-shaped loci traced on a daily basis, with considerable variation from one day to another, and approximately centred on the dip pole positions.

The location of the eccentric dipole, sometimes known as the magnetic centre, computed using the first eight Gauss coefficients for 2000.0 , is $(r, \theta, \lambda)=\left(540.56 \mathrm{~km}, 68.23^{\circ}\right.$, $\left.143.17^{\circ} \mathrm{E}\right)$.

An estimate of the accuracy of WMM2000 can be made for land locations by comparing observatory annual means and repeat station data collected from 1995.0 onwards with values computed from the model. These data have the effects of disturbance fields minimised but are contaminated by crustal fields. This results in considerable contributions from the crustal fields to the residuals. The results of these comparisons are given in Table 3. Residuals $>1000 \mathrm{nT}$ are excluded from the statistics.

The spatial distributions of these residuals show no significant patterns. One explanation for the observatories having higher residuals than the repeat stations is that a number of the observatories are on volcanic islands which have considerable crustal fields. Another is that there is more scope for selecting repeat station sites to be in areas with low crustal fields. In ocean areas the errors are expected to be less.

\section{Regional Modelling}

The main difference between regional and world magnetic models, is that the former characterise regional crustal magnetic anomalies as well as the core magnetic field. The first step in generating the regional models is to generate a high degree and order, $n i_{\max }=20$ in this case, spherical harmonic World Magnetic Model. Spherical harmonic degrees 13 to 20 contain the regional information. Other than the model degree, the procedure for generating this model is identical to that used to generate WMM2000.

The regional models for the continental United States, Alaska and Hawaii were produced by using the high degree and order global model to generate a $1^{\circ} \times 1^{\circ}$ grid at epochs
2000.0 and 2005.0 over each of these areas. Subsequently, spherical harmonic least-squares analysis was performed on each of the six grids. Secular-variation coefficients are obtained by differencing the results for the two epochs for each area. As the gridded data originated from a single model, each grid point was given the same weight, except for equalarea weighting and no Gaussian weight factors were necessary to down-weight outliers associated with disturbance field effects. The continental US was modelled to degree and order 6, Alaska to degree and order 5 and Hawaii to degree and order 3. Their Gauss coefficients along with their regional limits of validity are respectively given in Tables 4, 5, and 6.

The mean fits of each model with respect to their respective grids at epoch 2000.0 are essentially zero. The r.m.s. values with respect to these grids were $4.2 \mathrm{nT}$ for the continental US, $6.1 \mathrm{nT}$ for Alaska and $1.5 \mathrm{nT}$ for Hawaii.

Acknowledgments. The staff of magnetic observatories and survey organisations around the world are thanked for providing the data on which this model depends. The Ørsted satellite Overhauser data were kindly provided by the Danish Meteorological Institute. The preliminary $D s t$ indices were provided by the University of Kyoto, Japan and the $K p$ indices were provided by the Adolf-Schmidt-Observatorium für Geomagnetismus, GeoForschungsZentrum, Potsdam, Germany. Both indices are based on 1-minute data from many observatories. This paper is published with the permission of the Director, British Geological Survey (Natural Environment Research Council).

\section{References}

Lowes, F. J. and J. E. Martin, Optimum use of satellite intensity and vector data in modelling the main geomagnetic field, Phys. Earth Planet. Int., 48, 183-192, 1987.

Quinn, J. M., R. J. Coleman, D. L. Shiel, and J. Nigro, The Joint US/UK 1995 Epoch World Magnetic Model, Naval Oceanographic Office, Stennis Space Center, MS: Technical Report \#314, 1995.

Stern, D. P., R. A. Langel, and G. D. Mead, Backus effect observed by MAGSAT, Geophys. Res. Lett., 7, 941-944, 1980.

S. Macmillan (e-mail: S.Macmillan@bgs.ac.uk) and J. M. Quinn (e-mail: quinn@ghtmail.cr.usgs.gov) 